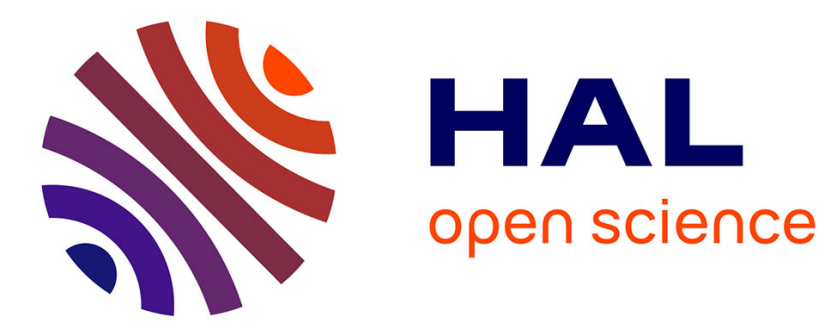

\title{
Deflections of inflatable fabric panels at high pressure
} Christian Wielgosz, Jean-Christophe Thomas

\section{To cite this version:}

Christian Wielgosz, Jean-Christophe Thomas. Deflections of inflatable fabric panels at high pressure. Thin-Walled Structures, 2002, 40 (6), pp.523-536. 10.1016/S0263-8231(02)00010-1 . hal-01006721

\section{HAL Id: hal-01006721 \\ https://hal.science/hal-01006721}

Submitted on 3 Dec 2016

HAL is a multi-disciplinary open access archive for the deposit and dissemination of scientific research documents, whether they are published or not. The documents may come from teaching and research institutions in France or abroad, or from public or private research centers.
L'archive ouverte pluridisciplinaire HAL, est destinée au dépôt et à la diffusion de documents scientifiques de niveau recherche, publiés ou non, émanant des établissements d'enseignement et de recherche français ou étrangers, des laboratoires publics ou privés.

\section{(ㄷ)(1)}

Distributed under a Creative Commons Attribution| 4.0 International License 


\title{
Deflections of inflatable fabric panels at high pressure
}

\author{
C. Wielgosz, J.-C. Thomas \\ Laboratoire de Génie Civil de Nantes-Saint Nazaire, Faculté des Sciences et des Techniques, \\ Université de Nantes, 2, rue de la Houssinière, BP 92208, 44322 Nantes Cedex 03, France
}

Inflatable structures made of modern textile materials with important mechanical characteristics can be inflated at high pressure (up to a several hundreds of $\mathrm{kPa}$ ). They can be used as strong building elements thanks to their mechanical strength. The aim of the paper is to present experimental and analytical studies on the behaviour of inflated fabric panels at high pressure and submitted to bending loads. It is shown that inflatable structures cannot be viewed as ordinary plates or beams, because their deformation pattern is quite different. Experiments show that their behaviour depends on the applied load, the inflation pressure, and the constitut-ive law of the fabrics. Equilibrium equations are written in the deformed state to take into account the influence of geometrical stiffness and the following forces. A Timoshenko's beam theory must be used because sections of the panels do not satisfy the usual Bernoulli's beam theory. A new inflatable beam theory is developed. Wrinkling loads are derived from equilib-rium equations. Deflections satisfy the fact that the compliance of the inflatable panel is the sum of the beam compliance and of the yarn compliance. Comparisons between the results of our modelling and experimental results are shown and prove the accuracy of this theory on the mechanical strength of inflatable structures at high pressure. 


\section{Introduction}

This paper presents results from research on the mechanics of inflatable structures at high pressure. Such structures are not new and a lot of inflatable structures are used by industry: temporary buildings, boats, pools, space antennas, life jackets, etc... Inflated structures have many interesting properties: they are light, easily folding and present reversible behaviour after 'failure' (they come back to their initial position after unloading). Inflation causes tension prestressing in the walls and in the yarns of the structures. This prestressing is proportional to the pressure and ensures an important mechanical strength. Generally, these structures are used with an inflation pressure lower than $100 \mathrm{kPa}$ because they are built with low strength materials. Although inflatable structures are not a recent development, no studies have been conducted on this subject for high pressure values. A few papers can be found on the behaviour of inflated tubes. Leonard et al. [1], Comer and Levy [2] and Main et al. [3] have studied the case of cantilever inflated fabric tubes at low pressure. They have shown that the wrinkling and/or collapse load is proportional to the applied pressure. This result has been verified at high pressure ([4], [5]) and comparisons between theoretical and experimental wrinkling loads of panels and tubes show a good accuracy between theory and experiments. We must therefore use a high pressure if we want a good mechanical strength of inflatable structures.

Unfortunately there are few results on the deflections of inflatable structures. Main et al. [3] have studied inflatable cantilever fabric tubes and calculated the deflections of these structures by using the usual beam theory. Experimental and theoretical results were compared, and they have proved that this theory can be applied to inflatable tubes. In fact they have used fabrics with low characteristics materials (20 times lower than our fabrics) and their study was done at a pressure almost five times lower that the scale which is used here. We think that these studies should be extended. The obvious reason is that in a beam theory, the values of the deflections depend only on the flexural rigidity of the beam. The pressure does not appear in the beam solution, and it seems clear that an inflated beam at an extremely low pressure can have very large deflections. We have tried to apply a yarn theory to inflatable panels and tubes ([4], [5]) and in this case the result is in reverse order: the deflection values are close to experimental ones when the pressure is low, but deviate greatly from reality at high pressure. Moreover, in a yarn theory deflections are independent of the material constitutive law and this is irregular. The only way to obtain good results on the deflection values was to use numerical modelling, but the studies were tedious ([4], [6]). We will show that in fact the deflections of inflatable structures submitted to bending loads are a linear set of yarn and beam deflections. Given that these deflections are inversely proportional to the constitutive law and to the applied pressure, we have a second reason to greatly inflate the structures and to use high characteristics materials.

In the first section of the paper we will describe experimental work and show that inflatable panels cannot be viewed as ordinary plates or beams, because their deformation pattern is quite different. Experiments show that they behave like yarns when the pressure is low, like beams at high pressure and once again like yarns or 
mechanisms when the applied load is near their wrinkling load. A new inflated beam theory is therefore constructed in the second section in order to provide simple analytical formulas for the wrinkling load and for the deflection values of simply supported inflated panels submitted to inflation pressure going up to $300 \mathrm{kPa}$. Equilibrium equations are written in the deformed state to take into account the geometrical stiffness of the beam and the following forces. A Timoshenko's beam theory is used because sections of the panels do not satisfy the usual Bernoulli's beam theory. It will be shown that the compliance of inflated fabric beams is only the sum of yarn compliance and beam compliance. Analytical results are close to experimental ones.

\section{Experimental results on inflatable panels}

The tested panels are prototypes constructed by Tissavel Inc. They are made of two parallel-coated woven fabrics connected by yarns and a cross section of these panels is shown in Fig. 1. The yarn density is enough to ensure the flatness of the fabric structure. Fabrics are made with high strength polyester.

The panel's behaviour depends on the inflation pressure $p$ that leads the fabrics and the yarns to be prestressed and then to support local compression loads. The panels are simply supported beams loaded by a concentrated force $F$. The width and height are $b, h$ and $l$ is the half span of the beam. The shapes of the deformed panel are shown in Fig. 2 for two values of the inflation pressure (50 and $300 \mathrm{kPa}$ ).

One can see that the bending shape depends on the pressure. When the pressure is low, large straight parts appear between points of loading and the free ends of the panel remain almost straight. When the pressure is higher, straight parts are shorter and a wider curved zone appears. In both cases we also take notice of light discontinuities of the rotation near loading areas (supports and loading point). This will be called local effects. The panel therefore has a behaviour that is similar to that of a tensioned yarn when the pressure is low and to that of a beam when the pressure is high. Experimental deflection curves for low and high values of the

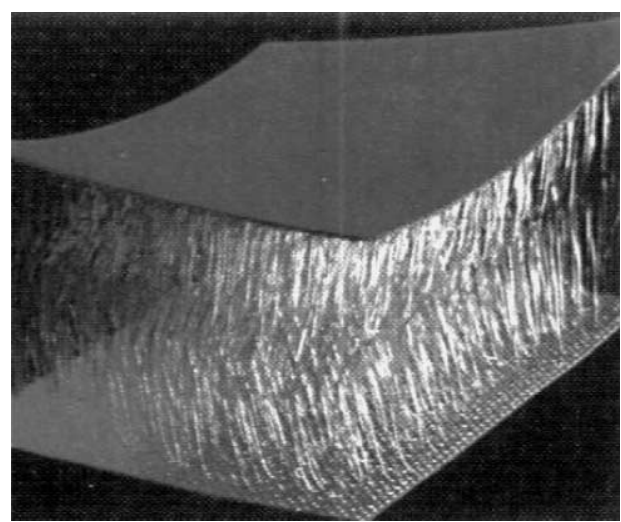

Fig. 1. Cross section of the panels 

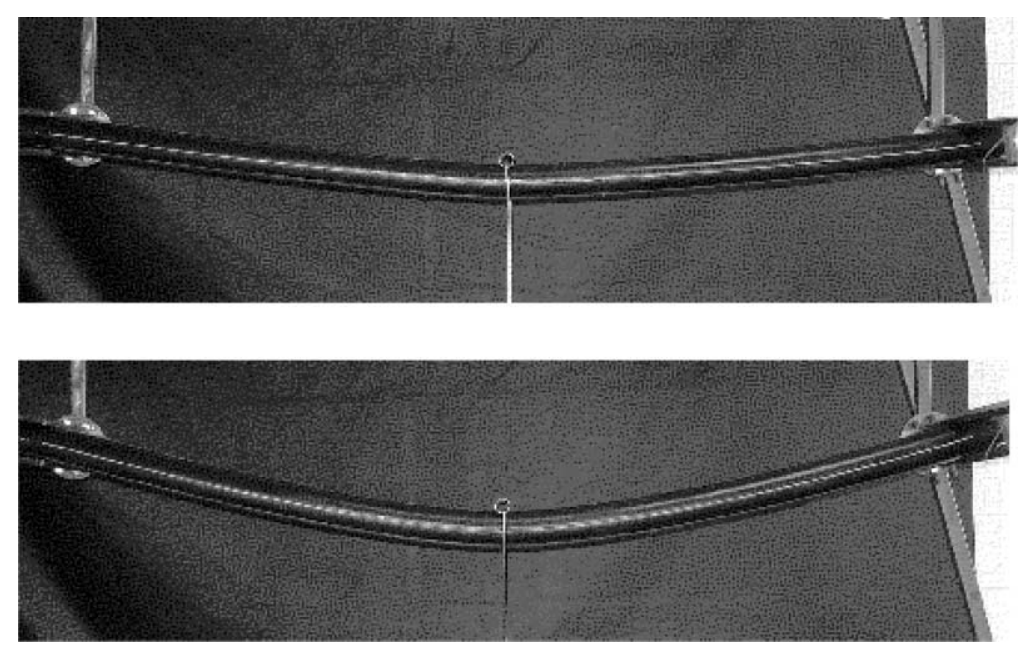

Fig. 2. Deformed pattern of inflated panels

inflation pressure are shown Fig. 3. When the load applied to the panel is near to its wrinkling or limit load, the shape is once again similar to that of a tensioned yarn as shown in Fig. 4.

All these experimental results prove that our inflatable panels behave successively like yarns and beams, and that their deformation pattern depends mainly on the inflation pressure and on the applied load.

From an engineering point of view, we must answer the following two questions: what is the limit load of an inflatable panel, and what is the maximum value of the deflection for a given load? We have shown [4] that we can easily answer the first question. Theoretical limit loads can be calculated with limit analysis because the deformation pattern at the limit load is similar to the yield mechanisms of beams. We have compared theoretical and experimental limit loads for the panels. Theoreti-

\section{Experimental deflection of an inflated panel}

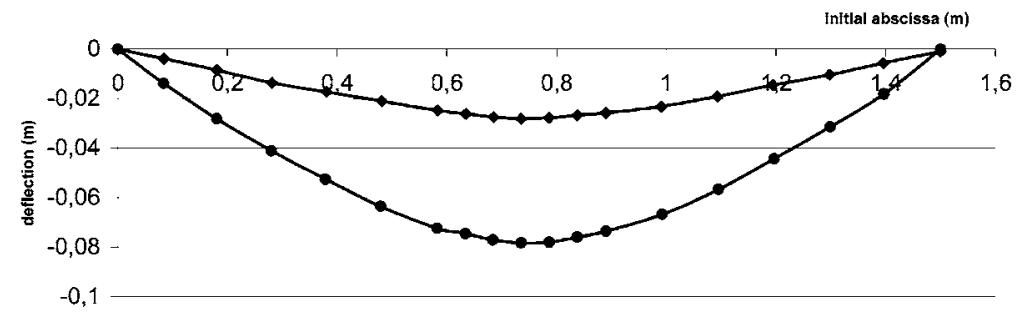

\section{$\rightarrow-50 \mathrm{kPa} 27 \mathrm{~N} \rightarrow-300 \mathrm{kPa}$-150N}

Fig. 3. Deflection curves for low and high pressure 


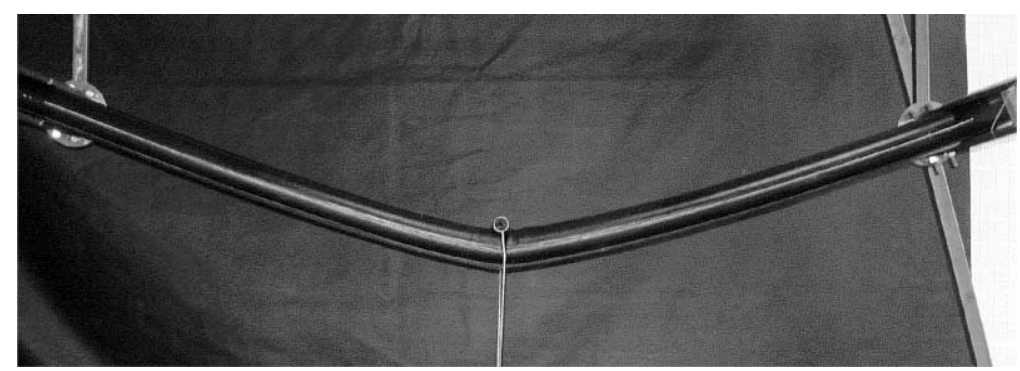

Fig. 4. Shape of an inflatable panel at its limit load (300 kPa)

cal values of the limit load are close to experimental results and are obtained within $20 \%$ error.

Unfortunately we have not yet found an easy answer to the second question. Some comparisons on the maximum values of the deflection at low pressure with the tensioned yarn theory were given in [4]. Theoretical values are close to experimental ones only for pressures lower than $50 \mathrm{kPa}$. Elsewhere a beam theory is not accurate because of the decreased curvature of the panels (where straight lines appear). In these areas the curvature is near zero and the bending momentum is linear; we cannot define a flexural rigidity. The second argument, which prevents the use of a usual beam theory, is due to the fact that in a beam theory the pressure is not taken into account. The only way to obtain correct values on the deflection was to use numerical modelling [4], but the computation times were tedious, because we had to take into account large displacements and following forces.

We will now develop the mechanical theory of inflatable panels. This theory is inspired by our experiments, and we will consider the panel as a Timoshenko'beam. Fig. 5 presents a zoom on the section's rotation, and one can see that the section is not orthogonal to the neutral fibre. We will show that we are able to explain all these behaviours of the panels and give simple analytical formulae for the deflections.

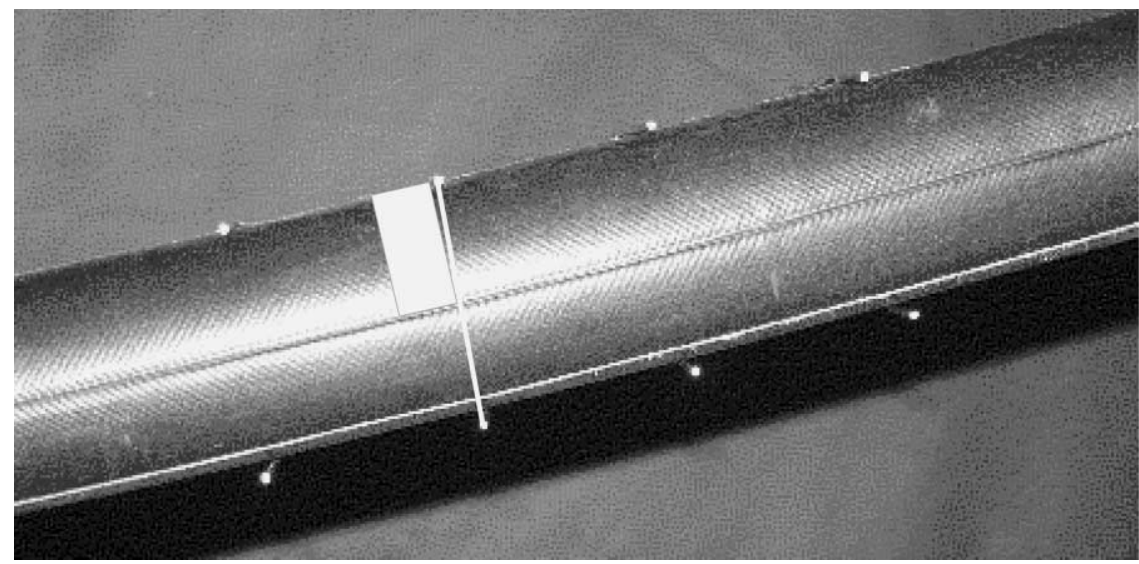

Fig. 5. Rotation of the section 


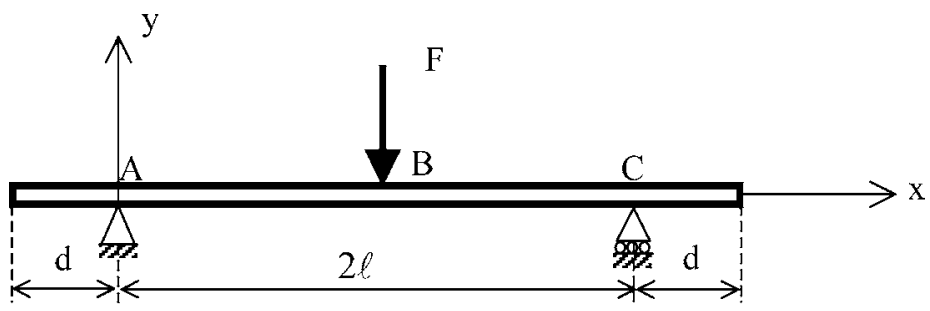

Fig. 6. Panel studied

\section{Mechanics of inflatable panels}

The mechanical strength of inflatable panels is obtained by use of its equilibrium equations, the constitutive law of the fabrics, and kinematical assumptions on its deformation pattern.

The theory will be developed in the case of a simply supported panel, loaded by a concentrated force in the middle of the structure. Fig. 6 gives an outline of the studied problem with the main notations.

\subsection{Equilibrium equations: wrinkling load}

Let us consider a small element of the panel (Fig. 7) with length $\mathrm{d} x$, width $b$, and height $h$. Equilibrium equations are written for this element in its deformed position. The pressure effects are supposed to be replaced by forces normally applied to the membranes, because they are in fact following forces.

The angle between the membranes and the horizontal axis is named $\theta$ and $v$ is the deflection $(\theta=\mathrm{d} v / \mathrm{d} x) . N_{\mathrm{i}}$ and $N_{\mathrm{s}}$ denote the resultant stresses respectively in the lower and upper membrane; $T$ is the shear stress.

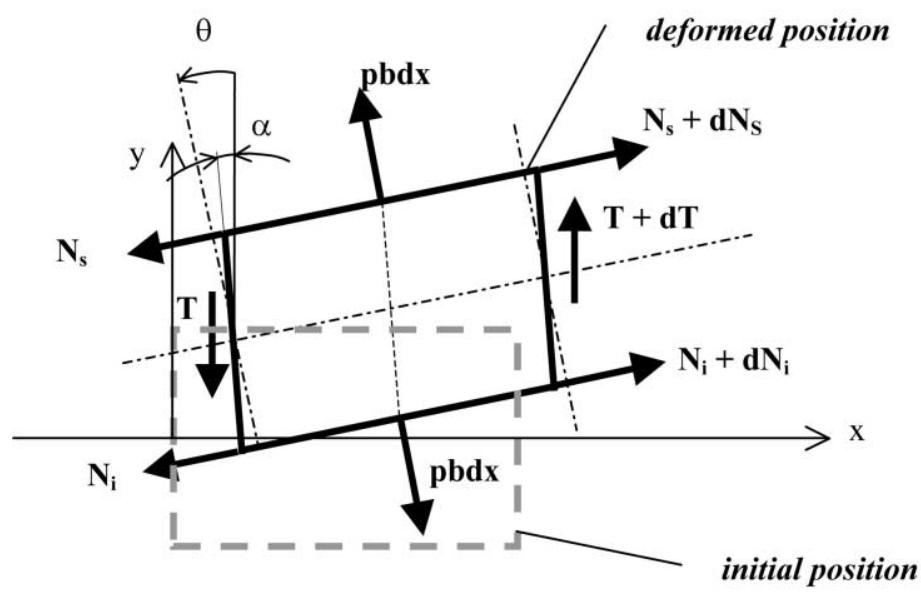

Fig. 7. Loads on an inflatable panel element 
We must use a Timoshenko's beam theory for this case of thin-walled structures, because the influence of the shear stress cannot be neglected, as in Bernoulli's beam theory. The straight section of the panel is supposed to remain straight after deformation, but not orthogonal to the neutral fibre, as seen in the last section. $\alpha$ denotes this rotation. All the theory is based upon the hypothesis that the structure's behaviour depends mainly on the values of $N_{\mathrm{i}}$ and $N_{\mathrm{s}}$.

Equilibrium equations are given by :

$$
\begin{aligned}
& \frac{\mathrm{d}\left(N_{\mathrm{i}}+N_{\mathrm{S}}\right)}{\mathrm{d} x} \cos \theta=0 \\
& \frac{\mathrm{d} T}{\mathrm{~d} x}+\frac{\mathrm{d}\left(N_{\mathrm{i}}+N_{\mathrm{S}}\right)}{\mathrm{d} x} \sin \theta=0 \\
& T+\frac{h \mathrm{~d}\left(N_{\mathrm{i}}-N_{\mathrm{S}}\right)}{2}-p b h \sin (\theta-\alpha)=0
\end{aligned}
$$

The first two equations show that $N_{\mathrm{i}}+N_{\mathrm{S}}$ and that the shear stress are constant.

The bending momentum results from the difference between the stresses in the two membranes :

$$
M=\frac{h}{2}\left(N_{\mathrm{i}}-N_{\mathrm{S}}\right)
$$

The third equation can also be written as $(\sin (\theta-\alpha)) \approx(\theta-\alpha)$ :

$$
T+\frac{\mathrm{d} M}{\mathrm{~d} x}-p b h(\theta-\alpha)=0
$$

One can notice the influence of the following forces in this equation.

Stresses are given by the global equilibrium of a sheet of the panel (Fig. 8). The

For $\ell<\mathrm{x}<2 \ell$ :

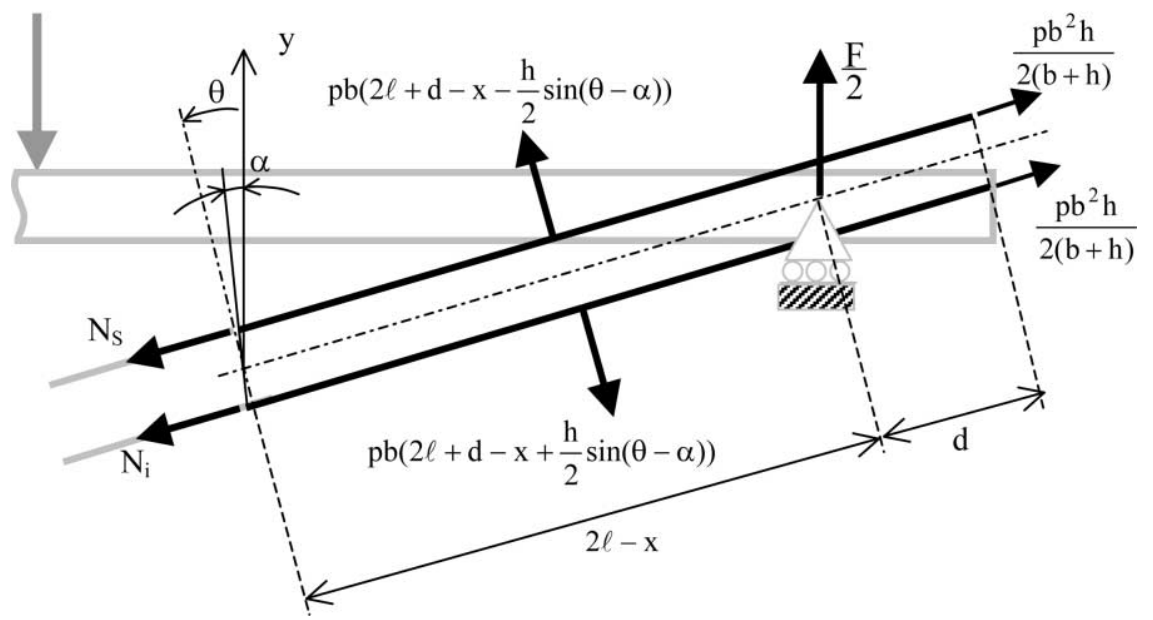

Fig. 8. Loads on a sheet of the panel 
equilibrium equation of the air included in the two membranes shows that the length of the lower membrane must be greater than the length of the upper membrane.

The equilibrium of this sheet gives the following equations:

$$
\begin{aligned}
& N_{\mathrm{S}}+N_{\mathrm{i}}=\frac{p b^{2} h}{b+h} \\
& T=\frac{F}{2}-p b h \sin (\theta-\alpha) \\
& h\left(N_{\mathrm{S}}-N_{\mathrm{i}}\right)+F(2 l-x)=0
\end{aligned}
$$

Eq. (6),(8) give the variation of the resultant stresses in the membranes :

$$
N_{\mathrm{S}}(x)=\frac{p b^{2} h}{2(b+h)}-\frac{F}{2 h}(2 l-x) \text { and } N_{\mathrm{i}}(x)=\frac{p b^{2} h}{2(b+h)}+\frac{F}{2 h}(2 l-x)
$$

The symmetry of the structure and its loading gives the graph (Fig. 9) of the theoretical membrane stresses in the panel.

These equilibrium equations show on the one hand that the difference of rotation terms $\theta-\alpha=\frac{\mathrm{d} v}{\mathrm{~d} x}-\alpha$ is constant, and on the other hand that the wrinkling load of the panel is given by (when the resultant stress in the upper fabric falls):

$$
F_{\mathrm{w}}=\frac{p b^{2} h^{2}}{l(b+h)}
$$

Let us now define the kinematics of the panel (Fig. 10). If $\mathrm{P}$ is a point of the neutral fibre, the displacement field is supposed to satisfy the following relation:

$$
\vec{u}(P)=u(x) \vec{e}_{x}+v(x) \vec{e}_{y}
$$

where the horizontal displacement $u(x)$ and the deflection $v(x)$ are only functions of $x$.

If $Q \mathrm{i}$ and $Q$ s are two points of the lower and upper membranes, their displacements are obtained by writing :

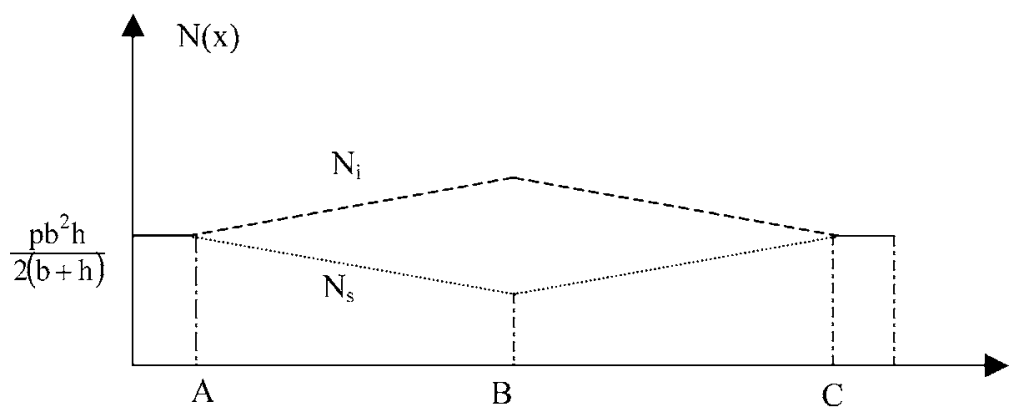

Fig. 9. Theorical membrane stress in the panel 


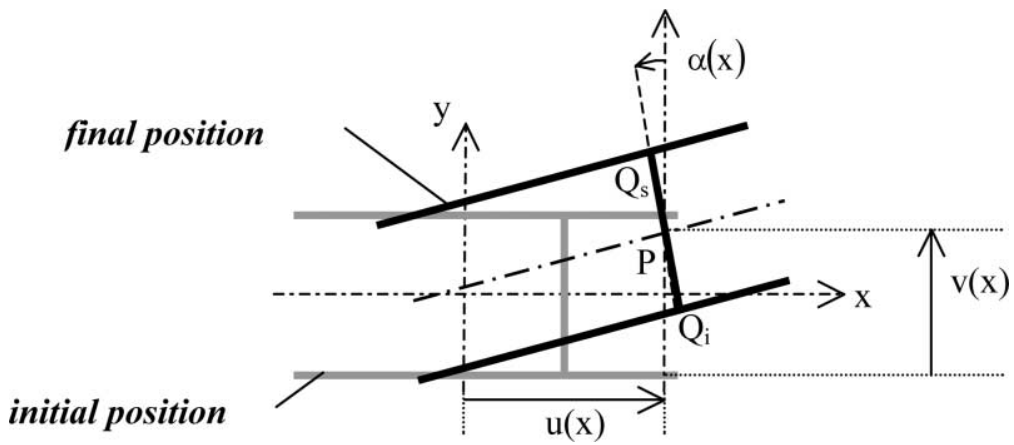

Fig. 10. Cross-section of the beam

$$
\vec{u}(Q)=\vec{u}(P)+\vec{\Omega} \wedge \overrightarrow{P Q} \quad \text { with } \quad \vec{\Omega}=\alpha \vec{e}_{z}
$$

The local strains $\varepsilon_{\mathrm{i}}(x)$ and $\varepsilon_{\mathrm{s}}(x)$ in the two membranes are therefore:

$$
\varepsilon_{\mathrm{i}}(x)=u_{, x}+\frac{h}{2} \alpha_{, x} \varepsilon_{\mathrm{s}}(x)=u_{, x}-\frac{h}{2} \alpha_{, x}
$$

Resultant stresses are obtained from the constitutive law of the fabric. A prestressed material satisfies the following law ( $\sigma_{0}$ is the pretension):

$$
\sigma=\sigma_{0}+E \varepsilon
$$

The resultant stresses in the membranes are therefore the following :

$$
N_{\mathrm{i}}(x)=\frac{p b^{2} h}{2(b+h)}+\frac{E^{*} b h}{2} \alpha_{, x} N_{\mathrm{s}}(x)=\frac{p b^{2} h}{2(b+h)}-\frac{E^{*} b h}{2} \alpha_{, x}
$$

where $E *$ is the membrane modulus (product of the Young's modulus $E$ by the thickness $e$ of the fabric).

The comparison between these formulas and the values providing from the equilibrium equations gives the differential equation satisfied by the rotation of a point of the neutral fibre of the panel:

$$
\alpha_{, x}=\frac{F}{E^{*} b h^{2}}(2 l-x)
$$

This is the usual beam solution :

$$
\alpha(x)=\frac{F}{E^{*} b h^{2}}\left(2 l x-\frac{x^{2}}{2}\right)+\alpha_{0}
$$

The boundary condition at the middle span of the panel implies that :

$$
\alpha(x)=\frac{F}{E^{*} b h^{2}}\left(2 l x-\frac{x^{2}}{2}-\frac{3 l^{2}}{2}\right)
$$


If we use the usual Timoshenko's theory, the relation between the shear stress and the rotation $\frac{\mathrm{d} v}{\mathrm{~d} x}-\alpha$ is written as:

$$
T=K G^{*} S^{*}\left(\frac{\mathrm{d} v}{\mathrm{~d} x}-\alpha\right)
$$

where $K$ is the shear coefficient, $G^{*}=G e, S^{*}=S / e$ and $G$ is the in-plane shear modulus of the panel.

Let us use Cowper's formulation [7] for a thin-walled box section with uniform thickness to calculate the shear coefficient. The graph presented in Fig. 11 shows the evolution of $K$ as a function of Poisson's ratio, for a panel with width $b=$ $0.2 \mathrm{~m}$ and height $h=0.055 \mathrm{~m}$.

We have also to consider the distribution of the shear stress in a section of the panel, and to take into account the influence of the connecting yarns, which transmit the greatest part of $T$. The yarns have been considered as stiff bars, with hinges at their tips, and their global behaviour produces a mechanism which ensures the flatness of the panel. The shear stress transmitted by the lateral walls is small compared to the shear stress which is transmitted by the yarns. We must add another coefficient $K^{\prime}$ :

$$
K^{\prime}=\frac{1}{(N+2)}
$$

where $N$ is the number of yarns in a section. We can write

$$
T=K K^{\prime} G^{*} S^{*}\left(\frac{\mathrm{d} v}{\mathrm{~d} x}-\alpha\right)=\frac{F}{2}-p b h\left(\frac{\mathrm{d} v}{\mathrm{~d} x}-\alpha\right)
$$

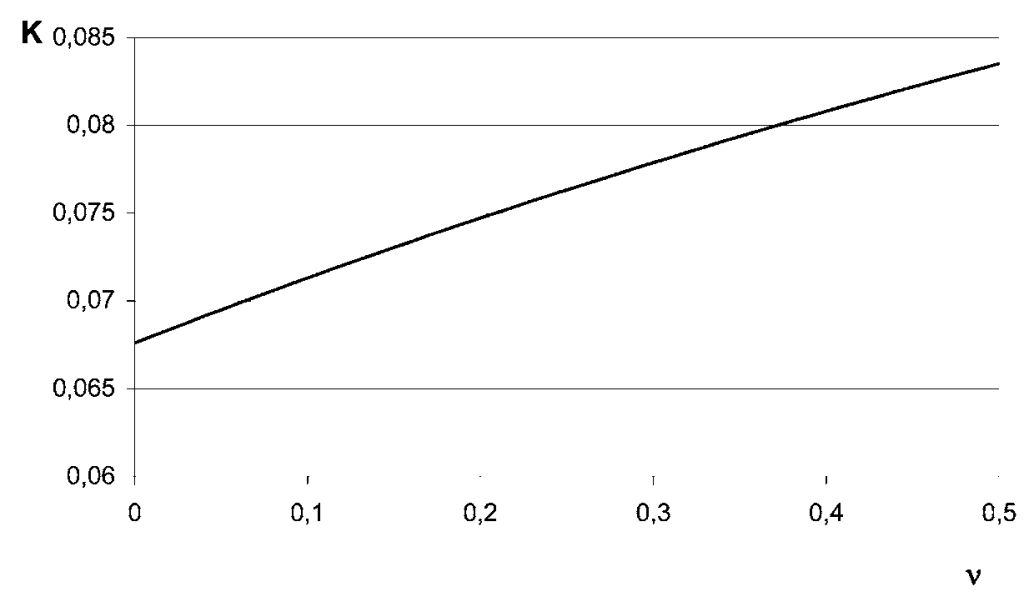

Fig. 11. Shear coefficient for a panel $(b=0.2 \mathrm{~m} ; \mathrm{h}=0.055 \mathrm{~m})$ 
So

$$
\frac{\mathrm{d} v}{\mathrm{~d} x}=\frac{F}{2\left(K K^{\prime} G^{*} S^{*}+p b h\right)}+\alpha
$$

By using the boundary conditions $v(2 l)=0$, we obtain the following formula for the deflection $l<x<2 l$

$$
v(x)=\frac{F}{2\left(K K^{\prime} G^{*} S^{*}+p b h\right)}(x-2 l)+\frac{F}{E^{*} b h^{2}}\left(-\frac{x^{3}}{6}+l x^{2}-\frac{3}{2} l^{2} x+\frac{l^{3}}{3}\right)
$$

This formula for the deflection of the panels can be compared to the result given by Fichter [8] for inflated cylindrical beams. A simple analogy between tubes and panels shows that we have the same kind of results. The way to solve the inflated structure behaviour is equivalent; Fichter used a variational formulation on tubes and we have used local equilibrium equations.

Let us now estimate the influence of the values of $K K^{\prime} G^{*} S^{*}$ and $p b h$. When the pressure is low $(100 \mathrm{kPa})$, the total load pbh equals $1100 \mathrm{~N}$. This load will increase with the pressure. Our panel presents almost 400 yarns $/ \mathrm{m}$. The shear modulus of the fabric is less than $200,000 \mathrm{~Pa} / \mathrm{m}$. In this case, the ratio between the values of $K K^{\prime} G^{*} S^{*}$ and $p b h$ is about $5 \%$. Our aim is to develop a theory for panels inflated at a greater pressure. So, we will suppose that:

$$
K K^{\prime} G^{*} S^{*}<<p h h
$$

With this approximation, the bending shape is finally given by $l<x<2 l$

$$
v(x)=\frac{F}{2 p b h}(x-2 l)+\frac{F}{E^{*} b h^{2}}\left(-\frac{x^{3}}{6}+l x^{2}-\frac{3}{2} l^{2} x+\frac{l^{3}}{3}\right)
$$

The same developpements applied to the first part of the beam leads to $0<x<l$

$$
v(x)=-\frac{F}{2 p b h} x+\frac{F}{E^{*} b h^{2}}\left(\frac{x^{3}}{6}-\frac{l^{2} x}{2}\right)
$$

And the maximum value of the deflection is:

$$
v(l)=-\frac{F}{2 p b h} l-\frac{F \quad l^{3}}{E^{*} b h^{2} 3}
$$

which is only the sum between the tight yarn and the beam deflections. In an other word, the compliance of the inflatable panel is the sum of the beam compliance and of the yarn compliance.

This result is used to explain the behaviour of our panels. When the pressure is low and when the membrane modulus is high, the first expression of the right hand side of this equation is prevalent and the global behaviour is described by the tensioned yarn behaviour. When the pressure is increased, the influence of this expression decreases and the behaviour of the panel becomes a linear set of yarn and beam behaviours. Now when the membrane modulus is low, the influence of the yarn solution can be weak even when the pressure is low ([3]). 


\section{Comparisons between experimental and theoretical results}

\subsection{Results on the wrinkling loads}

Panels have been tested by applying displacements with an extremely low strain rate. A load cell gives the applied load to the panel. We suppose that the wrinkling load is reached when the load-deflection curve becomes non-linear. A comparison between experimental and theoretical values of the wrinkling loads is given in Table 1. We can see that theoretical values are given with an error of approx. $10 \%$.

\subsection{Results on the deflection}

The wrinkling loads depend only on the applied pressure and the geometry of the panels. We have seen that the deflections also depend on the constitutive law of the fabrics. A sheet of the membrane fabrics (without the connecting yarns and without the induction) has been submitted to axial loads and its response gives the membrane modulus. The results depend on the time of the experiment: the type of materials used in the conception of the panels have viscous properties. It is obvious that creep is not taken into account in our simplified theory for the modelling of these structures. Fig. 12 shows the evolution of the load when a displacement is applied to one of the panels.

We must also point out that the next value of the membrane modulus is obtained

Table 1

Theoretical and experimental values of the wrinkling load

\begin{tabular}{llll}
\hline Pressure & $100 \mathrm{kPa}$ & $200 \mathrm{kPa}$ & $300 \mathrm{kPa}$ \\
\hline Theorical wrinkling load & $59.3 \mathrm{~N}$ & $118 \mathrm{~N}$ & $178 \mathrm{~N}$ \\
Experimental wrinkling load & $60 \mathrm{~N}$ & $108 \mathrm{~N}$ & $160 \mathrm{~N}$ \\
\hline
\end{tabular}

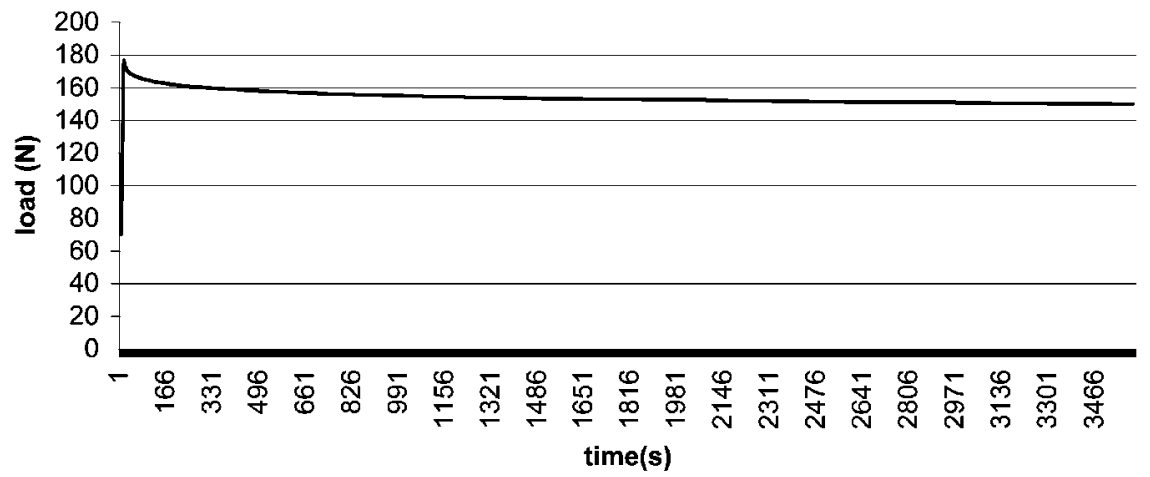

Fig. 12. Relaxation of the panel 
from data on a fabric sample at the same time used to present all our experimental results on the panels. In this case we obtained:

$$
E *=650,000 \mathrm{~Pa} \mathrm{~m}
$$

Fig. 13 shows the comparisons between theoretical and experimental values of the deflections of one of the panels for four values of the pressure (varying from 50 to $300 \mathrm{kPa}$ ).

\section{Conclusion}

It is advisable to use modern textile materials with high mechanical characteristics for imagining the behaviour of inflatable beams at high pressure. The paper deals with the first experimental and analytical results on modelling of the behaviour of inflatable fabric panels. The main results are relative to the wrinkling loads and the deflections under bending loads. Equilibrium equations are written in the deformed state to take into account the geometrical stiffness and the following forces. A Timoshenko's beam theory must be used because sections of the panels do not satisfy the usual Bernoulli's beam theory. Wrinkling loads are obtained from equilibrium equations. Inflatable panels cannot be viewed as ordinary beams, because their deformation pattern is a set of tensioned yarn and beam behaviour. A new inflatable beam theory has been developed and comparisons between experimental and theoretical results prove the accuracy of this theory on the mechanical strength of inflatable panels at high pressure. Development of new types of structures making full use of performance characteristics of modern textile materials is possible because that theory of their behaviour is now available when the pressure reaches several hundred

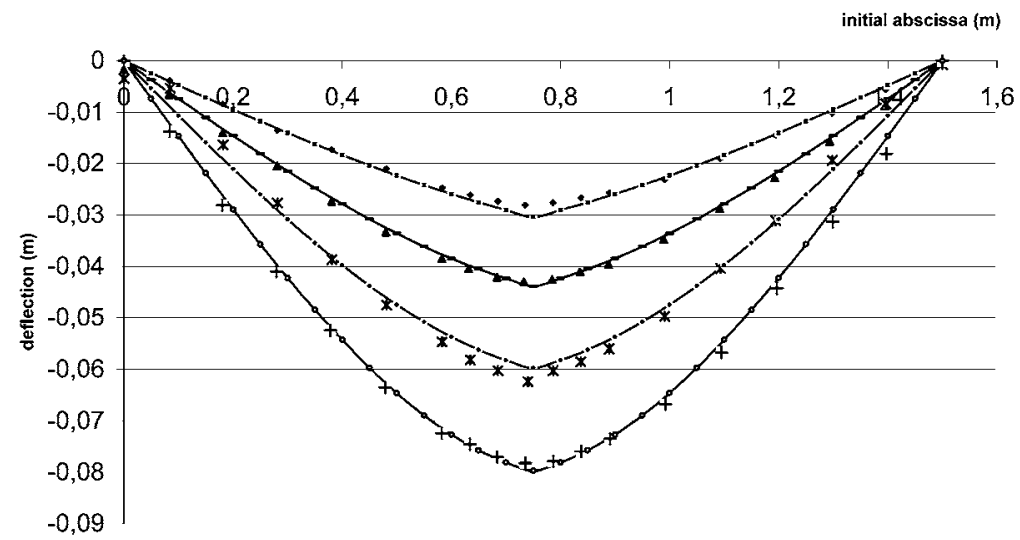

- Exp_50kPa_27N -- Th_50kPa_27N Exp_100kPa_57N $\longrightarrow$ Th_100kPa_57N

x Exp_200kPa_101N -.TTh_200kPa_101N + Exp_300kPa_150N $\rightarrow-T h \_300 k P a \_150 \mathrm{~N}$

Fig. 13. Theoretical and experimental values of the deflection 
$\mathrm{kPa}$. It is also possible to foresee the building of light, easily transportable and extremely strong fabric structures. Work is now required on the reliability of such structures in order to prove that they can be used by industry. Our results must be extended to other types of beams such as tubes. The influence of shear stresses must be improved. This paper is only concerned with isostatic beams. Work is in progress on the behaviour of hyperstatic panels and tubes.

\section{Acknowledgements}

The authors would like to thank Tissavel Inc for providing the panels and M. E. Leflaive for helpful discussions.

\section{References}

[1] Leonard RW, Brooks GW, McComb Jr, HG. Structural considerations of inflatable re-entry vehicles. NASA TN D-457, 1960.

[2] Comer RL, Levy S. Deflections of an inflated circular cylindrical cantilever beam. AIAA Journal 1963;1(7):1652-5.

[3] Main A, Peterson SW, Strauss AM. Load-deflection behaviour of space-based inflatable fabric beams. Journal of Aerospace Engineering 1994;2(7):225-38.

[4] Wielgosz C, Leflaive E, Dubé JF. Experimental study and numerical modelling of inflated fabric panels. In: Hoa V, De Wilde WP, Blain WR, editors. Computer methods in composite materials VI. Computational Mechanics Publications; 1998. p. 137-45.

[5] Wielgosz C, Leflaive E, Dubé JF, Thomas JC. Behaviour of inflated fabric beams at medium pressures. In: Proceedings of the Twelve International Conference on Composite Material. I.C.C.M. 12, 1999.

[6] Wielgosz C, Leflaive E, Thomas JC. Behaviour of inflated fabric structures at medium pressures. In: Proceedings of the Third International Conference on Mobile And Rapidly Assembled Structures. MARAS 2000, Madrid, 21-23 June 2000: 137-45.

[7] Cowper GR. The shear coefficient in Timoshenko's beam theory. Journal of Applied Mechanics 1967;33:335-40.

[8] Fichter WB. A theory for inflated thin-wall cylindrical beams. NASA TN D-3466, 1966. 